\title{
Improving Ergonomics in Teleoperation
}

\section{Muñoz LM ${ }^{*}$}

Automatic Control and Computer Engineering Department, Universitat Poletecnica de Catalunya, Spain

*Corresponding author: Muñoz LM, Automatic Control and Computer Engineering Department. Universitat Poletecnica de Catalunya, Spain, Tel: 938967231; E-mail: luis.miguel.munoz@upc.edu

Rec date: Nov 11, 2014, Acc date: Nov 12, 2014, Pub date: Nov 25, 2014

Copyright: $\odot 2014$ Muñoz LM. This is an open-access article distributed under the terms of the Creative Commons Attribution License, which permits unrestricted use, distribution, and reproduction in any medium, provided the original author and source are credited.

\section{Editorial}

Human-machine interaction in teleoperation, through the Automatic Control and Computer Engineering Department, Universitat Poletecnica de Catalunya adequate user interface, allows achieving the level of intelligence necessary to execute complex tasks that cannot be executed by machines or robots alone neither directly by humans. Human-Robot interaction techniques facilitate the execution of such tasks making them more efficient and effective through the improvement of their user interface.

Humans have inherent motor limitations (such as physiological tremor) and perceptive limitations (mainly perception of distance and time), which can prevent them from operating smoothly and precisely enough for certain applications. Some studies have already tackled this problem and its effect on the human-machine interaction and teleoperated systems. There are psychomotor models that show that the human manipulation efficiency, in actions such as pointing an object, depends on several factors. Among these models, the most representative corresponds to Fitts' Law [1], in which the execution time is a logarithmic function of the size and distance to the object.

In teleoperation, and based on these models, a modification of the visual scale in the user's interface has a direct effect on the task execution time and on the precision that can be achieved. The same occurs with a change in the amplitude of the movement executed by the human operator with respect to that performed by the system. Therefore, scaling the movement between master and slave has a significant effect on the efficiency and effectiveness executing a task.

Recent research $[2,3]$ is oriented to the design and development of methods conceived to improve effectiveness thanks to a larger visual and motor efficiency of the human-machine interface. These methods are based on the modification of the information flow between human, machine and interface by means of the scaling of both, the human movements and the image of the visualized task. Operation time, hand movements and the need for visual attention can thus be reduced with this computerized assistance. The changes of scale adapt to the task, which positively affects its performance in terms of precision, speed and ergonomics.

Therefore, these methodology aims to link the human operator working space to the machine or robot working space through an interface that introduces two scaling processes. A first change of scale is applied between the movement produced by the human operator and the movement produced in the visual interface (for instance, movement of the robot end-effector that is visualized on the computer screen); and a second change oriented to scale the real space of the task over the visual space of the interface.

These changes of scale should be adjusted to the objects of interest, which result in a modification of the spatial resolution according to the task to be performed and to the size, shape distance and speed of the objects. Such changes modify the information flow between human and machine according to the characteristics and limitations of both.

\section{References}

1. Fitts PM (1954) The information capacity of the human motor system in controlling the amplitude of movement. J Exp Psychol 47:381-391.

2. Munoz LM, Casal, A, Frigola M, Amat, J (2011) Motor-Model-Based Dynamic Scaling in Human-Computer Interfaces. IEEE Trans Syst Man Cybern B Cybern 41:435-447.

3. Munoz LM, Casals A (2012) Dynamic Scaling Interface for Assisted Teleoperation. IEEE International Conference on Robotics and Automation, Saint Paul, MN, USA, pp. 4288-4293. 